\title{
Liver and Spleen Injuries and Associated Rib Fractures: An Autopsy Study
}

Nuwadatta Subedi* ${ }^{*}$, Yadav $\mathrm{BN}^{2}$ and Shivendra $\mathrm{Jha}^{2}$

${ }^{1}$ Department of Forensic Medicine, College of Medical Sciences, Bharatpur, Chitwan, Nepal

${ }^{2}$ Department of Forensic Medicine, BP Koirala Institute of Health Sciences, Dharan, Nepal

\begin{abstract}
Extra thoracic injuries in rib fractures often include intra-abdominal organ injuries, commonly liver and spleen. The study was conducted with an objective to determine the association between rib fractures and liver and spleen injuries among medico legal autopsies with blunt abdominal trauma at the mortuary of tertiary referral centre in eastern Nepal for the period of one year.

Among a total of 68 cases studied, male: female ratio was 3.53:1 and the mean age $30.76 \pm 5.14$ years. The presence of liver injuries was significantly increased in all rib fractures (P value 0.378 , OR 1.552) and right sided only or bilateral ribs fractures ( $P$ value $<0.05$, OR 0.33 ). Spleen injuries are significantly associated with left sided only or bilateral rib fractures ( $P$ value $<0.05$, OR 3.02).
\end{abstract}

A significant difference exists between organ injuries with associated rib fractures compared to those without fracture, implying that the presence of rib fracture is a good indicator of organ injury.

Keywords: Autopsy; Liver injury; Rib fractures; Spleen injury

\section{Introduction}

The abdominal cavity extends superiorly into the osseocartilaginous thoracic cage to the fourth intercostal space. Consequently, the more superiorly placed abdominal organs (spleen, liver, part of the kidneys, and stomach) are protected by the thoracic cage [1]. It should be appreciated that, from the forensic aspect, the spleen and most of the liver and stomach are thoracic organs in that they lie largely beneath the costal margin, and are vulnerable to both stabbing and blunt injury to the chest [2]. Liver is the largest gland in the body. The anterior surface is triangular and is related to the xiphoid process and the diaphragm on either side. Owing to the size, its fixed position and friable consistency, the liver is frequently wounded by a stab in the abdomen, is often ruptured by a blunt trauma. It may also be lacerated by the fractured ends of the ribs perforating the diaphragm. Spleen lies in the left hypochondriac region of the abdomen, its long axis being parallel to that of $9^{\text {th }}$ rib, behind the stomach and inferior to the diaphragm. On account of its position, rupture of the spleen is rare unless caused by considerable crushing or grinding force. The spleen may sometimes be ruptured by the broken ends of a rib which may be caused by the blunt trauma [3].

Although patterns of injuries may appear obvious on the skin, examination of the distribution of fractures and visceral injuries may be the only way to understand the events during the accident. Examination of the internal injuries may be the only means of documenting the mechanics of their death [4]. Associated extrathoracic injuries in rib fractures, other than pulmonary complications, often include intraabdominal organ injuries; the most common of which include liver and spleen injuries [5].

The primary mechanism of chest and abdomen injury is compression of the body at high rates of loading. This causes deformation and stretching of internal organs and vessels. When the compression of the torso exceeds the ribcage tolerance, fractures occur and internal organs and vessels can be contused or ruptured. In some chest impacts, however, internal injury occurs without skeletal damage. This can happen during high-speed loading. It is due to the viscous or rate-sensitive nature of human tissue as biomechanical responses differ for low- and high-speed impact [3].
Clinical suspicion for classifying risk factors that increase the possibility of intra-abdominal organ injury in blunt traumatic rib fracture patients is extremely important. This is because such injuries can be easily overlooked as the signs are not clearly shown due to bleeding in the early phase of trauma due to volume of abdominal cavity, and thoracic trauma patients and relevant clinical symptom can be easily masked [6]. Early discovery of patients with rib fracture and intra-abdominal organ injury is extremely important to the prognosis. No similar studies from Nepal have been published so far. The study was conducted with the rationale to determine the relationship of liver and spleen injuries with overlying rib fractures so that it can be implied into day to day clinical and autopsy practice. This study was conducted with the objective of determination of the association between rib fractures and liver and spleen injuries among fatal blunt abdominal trauma cases.

\section{Materials and Methodology of the Study}

It is a hospital based cross sectional study. Materials for the present study were collected from the fatal cases with blunt abdominal trauma that underwent medico-legal autopsies at the mortuary of a tertiary hospital of Eastern Nepal during the period from 14 $4^{\text {th }}$ April 2010 to $13^{\text {th }}$ April 2011. A total of 68 cases were studied. The association of the liver and spleen injuries with rib fractures was studied in those cases.

All the autopsies showing the evidence of external or internal abdominal injuries caused by blunt mechanical trauma were included and the decomposed bodies were excluded from the study. The association of liver and spleen injuries was studied with the presence

*Corresponding author: Nuwadatta Subedi, Department of Forensic Medicine College of Medical Sciences, Bharatpur, Chitwan, Nepal, Tel: +977 9855054563 ; E-mail: subedind@yahoo.com

Received July 08, 2014; Accepted August 30, 2014; Published September 06 2014

Citation: Subedi N, Yadav BN, Jha S (2014) Liver and Spleen Injuries and Associated Rib Fractures: An Autopsy Study, J Forensic Res 5: 240 doi:10.4172/2157-7145.1000240

Copyright: ( 2014 Subedi N, et al. This is an open-access article distributed under the terms of the Creative Commons Attribution License, which permits unrestricted use, distribution, and reproduction in any medium, provided the original author and source are credited. 


\begin{tabular}{|c|c|}
\hline Cause of trauma & Number (Percent) \\
\hline Road traffic accidents & $63(92.6)$ \\
\hline Fall from height & $4(5.9)$ \\
\hline Assault by blunt weapon & $1(1.5)$ \\
\hline Total & $68(100)$ \\
\hline
\end{tabular}

Table 1: Cause of Trauma.

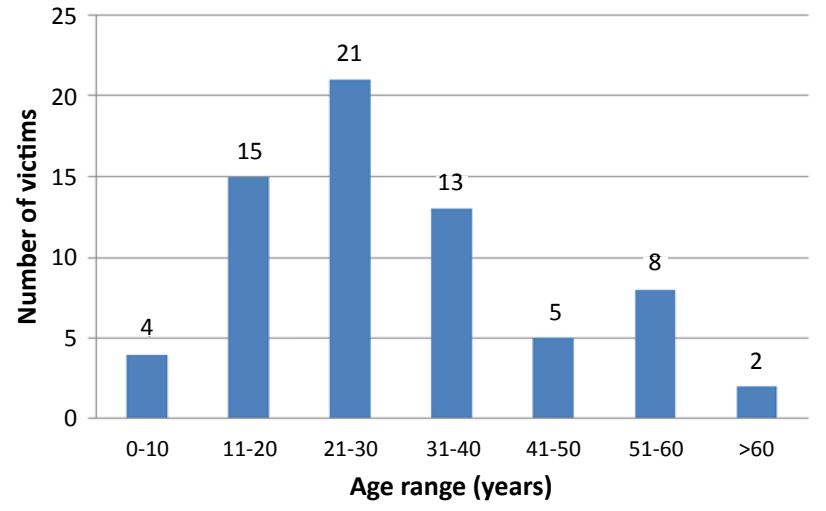

Figure 1: Age group of the victims.

\begin{tabular}{|c|c|c|c|c|c|}
\hline \multirow{2}{*}{ Liver } & & Fracture & No Fracture & P value & Odds Ratio \\
\cline { 2 - 5 } & Injury & $70.7 \%(29)$ & $39.3 \%(12)$ & & \\
\multirow{2}{*}{ Spleen Injury } & $40.7 \%(11)$ & $59.3 \%(16)$ & $<0.05$ & 3.515 \\
\cline { 2 - 4 } & Injury & $63.0 \%(17)$ & $37.0(10)$ & & \\
\cline { 2 - 4 } & No Injury & $56.1 \%(23)$ & $43.9 \%(18)$ & 0.317 & 1.330 \\
\hline
\end{tabular}

Table 2: Relationship of liver and spleen injuries with all rib fractures.

\begin{tabular}{|c|c|c|c|c|c|}
\hline & & Fracture & No Fracture & P value & Odds Ratio \\
\hline \multirow{2}{*}{ Liver } & Injury & $65.9 \%(27)$ & $34.1 \%(14)$ & & \\
\cline { 1 - 4 } & No Injury & $14.8 \%(4)$ & $85.2 \%(23)$ & $<0.005$ & 11.089 \\
\hline \multirow{2}{*}{ Spleen } & Injury & $29.6 \%(8)$ & $70.4 \%(19)$ & & \multirow{2}{*}{$0.330(-)$} \\
\cline { 2 - 5 } & No Injury & $56.1 \%(23)$ & $43.9 \%(18)$ & $<0.05$ & $0.30 \%$ \\
\hline
\end{tabular}

Table 3: Relationship of liver and spleen injuries with right sided only or bilateral rib fractures.

\begin{tabular}{|c|c|c|c|c|c|}
\hline & & Fracture & No Fracture & P value & Odds Ratio \\
\hline \multirow{2}{*}{ Liver } & Injury & $34.1 \%(14)$ & $65.9 \%(27)$ & & \\
\cline { 2 - 5 } & No Injury & $29.6 \%(8)$ & $70.4 \%(19)$ & 0.152 & 1.231 \\
\hline \multirow{2}{*}{ Spleen } & Injury & $48.1 \%(13)$ & $58.9 \%(14)$ & & \\
\cline { 2 - 6 } & No Injury & $21.9 \%(9)$ & $78.1 \%(32)$ & $<0.05$ & 3.02 \\
\hline
\end{tabular}

Table 4: Relationship of liver and spleen injuries with left sided only or bilateral rib fractures.

or absence of rib fractures. Rib fractures were categorised based on the side. The category of 'all rib fractures' includes any fractures irrespective of side. There were total 40 cases of rib fractures. 'Right side only or bilateral rib fractures' includes the fractures either on right side only or bilaterally $(\mathrm{n}=31)$ whereas 'Left side only or bilateral rib fractures' includes the fractures either on left side only or bilaterally $(n=22)$.

All collected data were compiled and entered into the Microsoft Excel and analysis was done using SPSS version 17.0. Chi square test was used to note the association of liver and spleen injuries with the ribs fracture categories. The probability of significance was set at $5 \%$ and $95 \%$ confidence limit. Ethical clearance was taken from the Institutional Ethical Committee of B.P. Koirala Institute of Health Sciences.

\section{Results}

Among a total of 68 fatal blunt abdominal trauma cases, 53 (78\%) of the victims were males and the rest $15(22 \%)$ were females. The most common cause of trauma was Road traffic accidents attributing to 63 (92.6\%) of the cases (Table 1). The age distribution is represented in Figure 1. Mean age was 30.76 years, with standard deviation of 15.14 .

Association of liver and spleen injuries with rib fractures was investigated in blunt trauma cases. Penetrating injuries were excluded as the mechanism of blunt trauma in different. Among 68 blunt abdominal trauma cases, liver was injured in $41(60.29 \%)$ and spleen in 27 (39.7\%) cases (Tables 2-4).

When all rib fractures were considered, it is evident that those with rib fractures have 3.515 times more chances of having liver injuries than without rib fractures ( $\mathrm{P}$ value $<0.05$ ). There is higher risk of spleen injuries in case of rib fractures but it is not significant ( $\mathrm{P}$ value 0.317 , OR 1.33) (Table 2).

When right sided only or bilateral rib fractures are considered, it was seen that the association of liver injuries with rib fractures is highly significant $(\mathrm{P}$ value $<0.005$, OR 11.089). Spleen injuries show significant negative association with right sided only or bilateral rib fractures $(\mathrm{P}$ value $<0.05$, OR -0.33 ) (Table 3 ).

If left sided only or bilateral rib fractures are taken into consideration, those with rib fractures have 3.02 times more chances of having spleen injuries than without rib fractures ( $\mathrm{P}$ value $<0.05)$. Risk of liver injuries is increased but it is not significant (P value 0.152, OR 1.231) (Table 4).

\section{Discussion}

The results of our analysis shows the patterns of liver and spleen injuries in relation to the presence or absence of overlying rib fractures. The significant association of liver and spleen injuries with ipsilateral rib fractures could be due to the direct transfer of energy and stretching of organs underneath the impact site. The fractured segments of the ribs can play a role. The vulnerability of liver injury in association of "all rib fractures" could be due to its large size so that the impact delivered even from the left side can cause its injury. Our findings are supported by Thor CP and Gabler HC [7], who had shown that in all types of crash mode, liver and spleen injuries are significantly associated with right sided only or bilateral rib fractures and left sided or bilateral rib fractures respectively. Ehyal Shweiki et al. [8] showed the probability of liver injury increased with the presence of any right-sided rib fracture, splenic injury with the presence of left sided rib fractures. Klinich et al. [9] presented that when all crashes are considered, liver injuries are significantly associated with any type of rib fractures except for those with lower left rib fractures and spleen injuries, with rib fractures on the left side. As presented by Al-Hassani A [10], Lower rib fractures, whether zone-limited or overlapping, are highly predictive of solid organ injury when compared with upper and mid zones. Multiple rib fractures involving the lower ribs have a high association with solid organ injury. Our study is unique as it exclusively includes fatal cases studied during autopsy. However as the sample size was less, the analysis of lower or upper rib fractures and side of impacts was not made as done by other studies $[7,8,11]$.

Extrathoracic injuries from rib fractures can be the result of a direct injury from the rib fractures, whether from the excess intrusion of the ribs or laceration from the fractured ribs. However, it is hard to analyze this relationship separately due to the difficulty in ascertaining whether the rib fractures and associated organ injuries are cause and effect in 
Citation: Subedi N, Yadav BN, Jha S (2014) Liver and Spleen Injuries and Associated Rib Fractures: An Autopsy Study. J Forensic Res 5: 240 doi:10.4172/2157-7145.1000240

Page 3 of 3

nature or merely due to a synergistic association [11]. A significant difference exists between organ injuries with associated rib fractures compared to those without fracture, implying that the presence of rib fracture is a good indicator of organ injury. So, whenever rib fractures are demonstrated in autopsy, presence of liver and splenic injuries have to be ruled out. It may be also implied in clinical practice where a test for checking abdominal injury must be conducted on such patients.

\section{Conclusion}

Among the fatal blunt abdominal trauma cases, presence of liver and splenic injuries was observed to have significant differences with those having rib fractures and those without them. The probability of presence of liver injuries is significantly increased in the cases having all rib fractures and right sided only or bilateral rib fractures, while the vulnerability of splenic injuries is significantly increased with left sided only or bilateral rib fractures. Our study implies that presence of rib fractures is a good indicator of underlying liver and spleen injuries.

\section{References}

1. Moore KL, Dalley AF (2006) Clinically Oriented Anatomy. (5th edn), Lippincott Williams \& Wilkins.

2. Abraham E, Cox M, Quincey D (2008) Pig-mentation: postmortem iris color change in the eyes of Sus scrofa. J Forensic Sci 53: 626-631.
3. Decker GAG (1986) Lee McGregor's synopsis of surgical anatomy. John Wright and Sons LTD, Bristol.

4. http://emedicine.medscape.com/article/1765532-overview

5. Shorr RM, Crittenden M, Indeck M, Hartunian SL, Rodriguez A (1987) Blunt thoracic trauma. Analysis of 515 patients. Ann Surg 206: 200-205.

6. Park S (2012) Clinical Analysis for the Correlation of Intra-abdominal Organ Injury in the Patients with Rib Fracture. Korean J Thorac Cardiovasc Surg 45: 246-250.

7. Thor CP, Gabler HC (2008) The relationship between thoracic organ injuries and associated rib fractures. Biomed Sci Instrum 44: 292-297.

8. Shweiki E, Klena J, Wood GC, Indeck M (2001) Assessing the true risk of abdominal solid organ injury in hospitalized rib fracture patients. J Trauma 50 : 684-688.

9. Klinich KD, Flannagan CA, Rupp JD, Sochor M, Schneider LW, et al. (2008) Fetal outcome in motor-vehicle crashes: effects of crash characteristics and maternal restraint. Am J Obstet Gynecol 198: 450.

10. Al-Hassani A, Abdulrahman H, Afifi I, Almadani A, Al-Den A, et al. (2010) Rib fracture patterns predict thoracic chest wall and abdominal solid organ injury. Am Surg 76: 888-891.

11. Flagel BT, Luchette FA, Reed RL, Esposito TJ, Davis KA, et al. (2005) Half-adozen ribs: the breakpoint for mortality. Surgery 138: 717-723. 